\title{
Increased Plasma Levels of the High Mobility Group Box 1 Protein (HMGB1) Are Associated With a Higher Score of Gastrointestinal Dysfunction in Individuals With Autism
}

\author{
K. BABINSKÁ ${ }^{1}$, M. BUCOVÁ ${ }^{2}$, V. ĎURMANOVÁ ${ }^{2}$, S. LAKATOŠOVÁ ${ }^{1}$, D. JÁNOŠÍKOVÁ \\ J. BAKOS̆ ${ }^{1,4}$, A. HLAVATÁ ${ }^{5}$, D. OSTATNÍKOVÁ ${ }^{1}$ \\ ${ }^{1}$ Institute of Physiology, Faculty of Medicine, Comenius University, Bratislava, ${ }^{2}$ Institute of \\ Immunology, Faculty of Medicine, Comenius University, Bratislava, ${ }^{3}$ Department of Psychology, \\ Faculty of Philosophy, Trnava University, Trnava, ${ }^{4}$ Institute of Experimental Endocrinology, \\ Slovak Academy of Sciences, Bratislava, ${ }^{5}$ Second Department of Pediatrics, Faculty of Medicine, \\ Comenius University and University Children's Hospital, Bratislava, Slovak Republic
}

Received March 16, 2014

Accepted August 6, 2014

\section{Summary}

Autism is a disorder of neural development characterized by impairments in communication, social interaction, restricted interests and repetitive behavior. The etiology of autism is poorly understood, the evidence indicates that inflammation may play a key role. In autism a high prevalence of gastrointestinal disturbances is reported, that are linked to a low-grade chronic inflammation of the intestinal mucosa. High mobility group box 1 protein (HMGB1) is an intranuclear protein that can be passively released from necrotic cells or actively secreted under inflammatory conditions as alarmin or late proinflammatory cytokine. The objective of this study was to measure plasma levels of HMGB1 in individuals with autism and to analyze their association with gastrointestinal symptoms. The study involved 31 subjects with low-functioning autistic disorder aged 2-22 years and 16 healthy controls. Plasma HMGB1 levels were significantly higher in individuals with autism than in controls (13.8 \pm 11.7 $\mathrm{ng} / \mathrm{ml}$ vs. $7.90 \pm 4.0 \mathrm{ng} / \mathrm{ml}, \mathrm{p}<0.02)$. In subjects with plasma HMGB1levels higher than $11 \mathrm{ng} / \mathrm{ml}$ severe forms of GI disorders were more prevalent $(83.3 \%)$ than in subjects with lower levels ( $38.9 \%, p<0.04)$. Results of the study support the involvement of the systemic low-grade inflammation in the pathomechanisms of autism and its possible association with GI symptoms.

\section{Key words}

Autism • Inflammation • HMGB1 plasma levels • Gastrointestinal symptoms

\section{Corresponding author}

K. Babinská, Institute of Physiology, Comenius University in Bratislava, Faculty of Medicine, Sasinkova 2, 81372 Bratislava, Slovak Republic. E-mail: katarina.babinska@fmed.uniba.sk

\section{Introduction}

Autism is a neurodevelopmental disorder defined by impairments across the areas of reciprocal social interaction, verbal and non-verbal communication, together with repetitive and stereotyped patterns of behavior and interests. The autism symptoms and their severity vary greatly and the wide range of conditions that are related to autism is collectively referred to as autism spectrum disorders (ASD). ASD are a lifelong disability that is manifested within the first 36 months of age. An increasing trend in incidence of ASD is observed worldwide (Elsabbagh et al. 2012), according to current data it affects 1 in 88 children, the prevalence being 4-5 times higher in boys than in girls (Centers for Disease Control and Prevention 2012). The etiology of autism is poorly understood, evidence shows that it is a multifactorial disorder, involving genetic, epigenetic and environmental factors.

Many of the cognitive and behavioral features of ASD are thought to arise from dysfunction of the central nervous system (CNS), however, evidence has documented multiple non-CNS abnormalities and 
comorbidities associated with ASD, such as gastrointestinal disorders, metabolic abnormalities, dysregulation of the immune functions and many others. It is suggested that ASD may arise from, or involve, also systemic abnormalities rather than being a purely CNS disorder, at least in a subset of individuals (Rossignol and Frye 2012). It was proposed that dysregulation of the immune system may be a key player in the pathophysiology of ASD (Depino 2013, Onore et al. 2012).

Recent research has provided evidence of how the immune system can profoundly impact neurodevelopment, cognitive functions, and behavior. Extensive alterations in immune function have been reported in ASD in which different mechanisms seem to play a role, all of them leading to a chronic state of low grade inflammation in the central nervous system. There is evidence indicating that immune responses in the periphery are also dysfunctional (Onore et al. 2012, Noriega and Savelkoul 2014).

Several studies revealed a higher prevalence of chronic gastrointestinal (GI) disturbances in subjects with ASD, such as diarrhea, constipation, abdominal pain and discomfort or gastroesophageal reflux. There is evidence that the underlying biology of GI disorders is linked to intestinal inflammation.

Levels of many immune markers have been shown to be altered in ASD. Collectively, these data reveal a trend towards pro-inflammatory immune activity and away from regulatory measures (Depino 2013, Onore et al. 2012).

The high mobility group box-1 protein (HMGB1) is a highly evolutionarily conserved and ubiquitous intracellular protein that when passively released out of cells or actively secreted has potent proinflammatory cytokine activity. HMGB1 has been implicated in several inflammatory and autoimmune disorders. In the gastrointestinal tract, increased levels of HMGB1 have been related to intestinal barrier dysfunction (Vitali et al. 2011), and in mouse models, to colonic inflammation (Splichalova and Splichal 2012). At the same time, HMGB1 has been shown to play role in neuroinflammation (Fang et al. 2012).

The existing scientific evidence indicates a possible involvement of HMGB1 in the multiple pathomechanisms of autism. The aim of this study was to determine the plasma HMGB1 levels in individuals with autism and to correlate them with the severity of gastrointestinal symptoms.

\section{Methods}

\section{Subjects}

The sample included 31 individuals (26 males and 5 females) with autism aged 2-22 years (mean age $9.0 \pm 5.6$ years). Criteria for inclusion involved diagnosis of severe autism (based on the ICD-10 criteria) with mental retardation (low-functioning autism), without neurological comorbidities, and without medication. The control sample involved 16 age matched controls (10 males and 6 females) aged 3-24 years (mean age $10.1 \pm 5.7$ years) including 10 siblings of the individuals with autism and 6 healthy volunteers, all without any psychiatric condition. Patients and controls did not significantly differ with regard to age $(p=0.55)$ and gender $\left(\chi^{2}=1.63, \mathrm{p}=0.20\right)$.

\section{Gastrointestinal symptoms}

These symptoms were assessed by a questionnaire. Information about occurrence of 6 types of GI disorders within the last 3 months was collected (abdominal pain, flatulence and bloating, diarrhea, constipation, pain in defecation, and voluminous feces). Frequency of the GI problems was rated on a scale from 0 to 4 ( 0 - free of the disorder, 1 - one to three times per month, 2 - once a week, 3 - several times per week, 4 daily). A score of GI disorders (GI score) was calculated for each individual as a sum of the ranks for all GI problems, the higher the score, the more severe form of the GI disturbance. Scores 5 or more were considered as a severe GI dysfunction.

\section{Laboratory methods}

Venous blood samples were drawn in the morning, centrifuged at $3000 \mathrm{~g}$ for $10 \mathrm{~min}$, and immediately after cell/serum separation frozen and stored at $-80{ }^{\circ} \mathrm{C}$ until analysis. HMGB1 levels were measured by a sandwich enzyme-linked immunosorbent assay (ELISA) (Shino-test Corporation, Japan / IBL Technology USA) according to the manufacturer's protocol.

\section{Data analysis}

Software GraphPad Prism 6 was used for the data analysis. Unpaired t-test with Welch correction was used for comparison of the mean values, and Chi-square test was used for comparison of categorical variables. Pearson's correlation coefficient was calculated to test the correlation between age and plasma HMGB1 levels. Results are presented as mean \pm standard deviation (SD). 
The Mann Whitney test was used for the comparison of the GI scores (due to their non-parametric distribution), and results are presented as medians and $95 \%$ confidence intervals. Values $\mathrm{p}<0.05$ were considered statistically significant.

The study was approved by the Ethical Committee of the Faculty of Medicine, Comenius University in Bratislava and an informed consent from all the subjects or their caregivers was obtained.

\section{Results}

The mean value of plasma HMGB1 levels (Fig. 1) was significantly higher in subjects with autism $(13.8 \pm 11.7 \mathrm{ng} / \mathrm{ml})$ than in the control group $(7.9 \pm 4.0$ $\mathrm{ng} / \mathrm{ml}, \mathrm{p}<0.02)$. No correlation of the HMGB1 plasma levels with age was found $(r=0.03 ; \mathrm{p}=0.86)$.

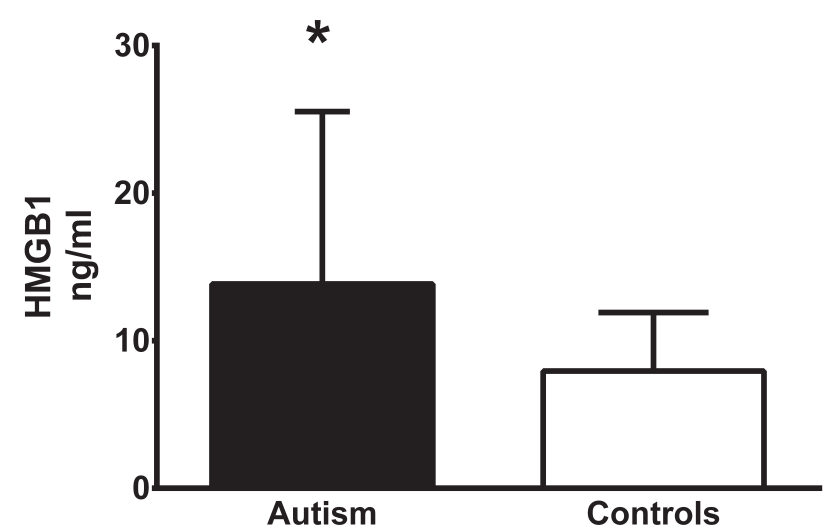

Fig. 1. Plasma HMGB1 levels (mean $\pm S D$ ) in individuals with autism and controls.

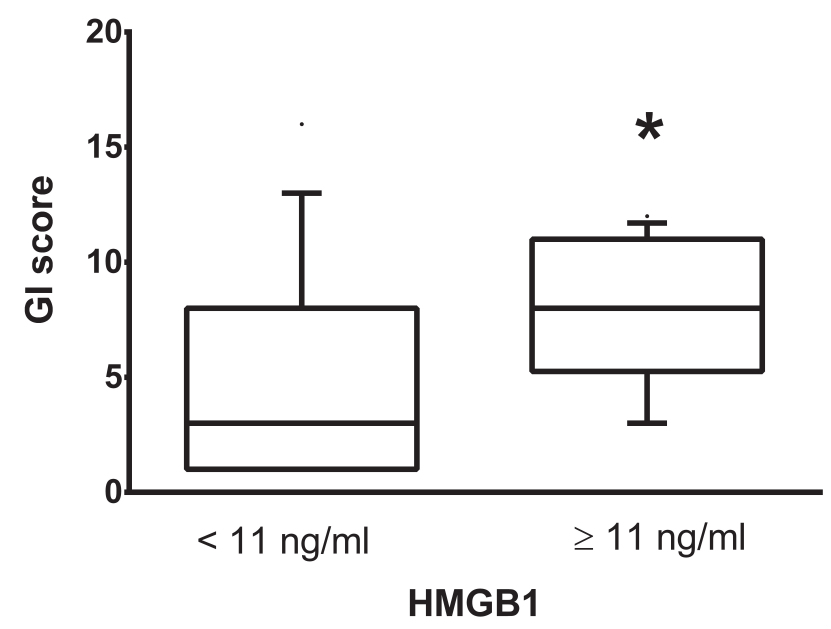

Fig. 2. Median values (and $95 \% \mathrm{CI}$ ) of the gastrointestinal dysfunction scores (GI score) according to plasma HMGB1 levels in individuals with autism (a higher GI score reflects a more severe form of GI disorder).
GI problems were found in $96.8 \%$ of subjects with autism, their prevalence $(66.6 \%)$ was significantly lower in the controls $\left(\chi^{2}=4.1, \mathrm{df}=1, \mathrm{p}<0.05\right)$. The score of GI symptoms in subjects with autism ranged from 0 (free of GI disorder, 1 individual) to 16 with median value 6.0 (95\% CI 3.0-8.0). In the subgroup of individuals with autism having plasma HMGB1 levels $11 \mathrm{ng} / \mathrm{ml}$ or higher $(n=19)$ a higher median score of GI symptoms was found $(8.0,95 \%$ CI 5.8-9.8). In the subgroup of patients with lower HMGB1 levels $(<11 \mathrm{ng} / \mathrm{ml}, \mathrm{n}=12)$ a lower median score of GI problems was observed (3.0, 95\% CI 2.9-7.2, $\mathrm{p}<0.04$, Fig. 2). Correspondingly, in the subgroup with higher HMGB1 levels $(\geq 11 \mathrm{ng} / \mathrm{ml})$ a significantly higher percent of subjects ( $73 \%$ ) suffered by more severe forms of GI problems (GI score 5 or more), whereas lower levels of HMGB1 $(<11 \mathrm{ng} / \mathrm{ml})$ were associated with lower prevalence of severe forms (37\%) of GI problems, and mild forms of GI symptoms prevailed in this group $\left(\chi^{2}=6.42, \mathrm{df}=1, \mathrm{p}<0.02\right)($ Fig. 3$)$.

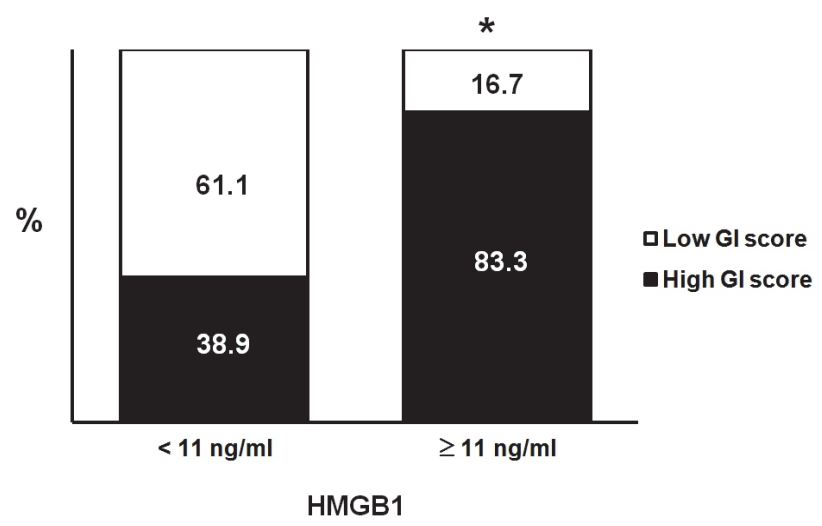

Fig. 3. Percent of subjects with a high GI score (severe GI symptoms) and low GI score (mild GI symptoms) according to the plasma HMGB1 levels in individuals with autism.

\section{Discussion}

Until recently, it was broadly accepted that genetic factors predominate in pathomechanisms of ASD, however recent studies of identical twins suggest, that there is a more substantial environmental component than previously believed (Hallmayer et al. 2011). A large number of environmental factors associated with ASD risk have been identified and it was proposed that the environmental challenges converge on some common pathways. One candidate that meets these requirements is the host inflammatory response (Depino 2013).

In ASD increased plasma levels of many proinflammatory cytokines have been reported, such as IL- 
1b, IL-6, IL-8 and IL-12p40 as well as MIF (macrophage migration inhibitory factor) and PDGF (platelet derived growth factor) (Onore et al. 2012). Aberrant expression of cytokines and their signaling intermediaries was observed in peripheral blood, also in the brain, and in the gastrointestinal tract in ASD patients (Goines and Ashwood 2013).

High mobility group box 1 (HMGB1) is an intranuclear non-histon protein widely expressed in cells of vertebrates. Extracellular HMGB1 is passively released from necrotic cells or actively secreted in response to proinflammatory stimuli, either infectious such as lipopolysaccharides, or non-infectious mediated through inflammatory cytokines TNF- $\alpha$, IL-1, IL-6, and IL-8, from a variety of immune cells or DAMPs (damage associated molecular patterns) released from damaged tissues or cells. It mediates inflammation by activating innate immunity through signal transduction in toll-like receptors and the receptor for advanced glycation endproducts (Fang et al. 2012, Naglova and Bucova 2012). However, HMGB1 appears to play double-edged roles, both during neural development and also in neurodegeneration. In early brain development, it facilitates neurite outgrowth and cell migration. During adulthood, it serves to induce neuroinflammation after injury.

Changes in HMGB1 levels have been observed in many neurological diseases, e.g. Huntington's disease, Alzeimer's disease, Parkinson's disease, and multiple sclerosis (Fang et al. 2012). Our literature survey revealed only two studies that assessed the HMGB1 levels in autism, both performed in small samples. Similarly to our results, Russo (2013) found elevated plasma levels of HMGB1 in ASD patients. Moreover, the plasma HMGB1 levels correlated with low levels of epidermal growth factor that is involved in growth and differentiation of cells in the CNS and gastrointestinal tract. Similarly, Emanuele et al. (2010) observed in a sample of young adults with autism higher HMGB1 concentrations than in controls together with an independent direct association between HMGB1 levels and the ADI-R (Autism Diagnostic Interview-Revised) social score: higher HMGB1 levels were associated with a worse social interaction.

Correspondingly, results of our study show higher HMGB1 levels in subjects with autism than in controls. Moreover, elevated plasma levels of HMGB1 are associated with higher severity of GI disorders in children and young individuals with low-functioning autism. The results support the hypothesis that inflammatory process may underlie the GI symptoms observed in some subgroups of subjects with autism. It has been documented in animal experiments that HMGB1 is associated with alterations in gut barrier function (Sappington et al. 2002). Recently, it was shown (Vitali et al. 2011) that HMGB1 is secreted by human inflamed intestinal tissues and abundantly found in the stools of patients with intestinal bowel disease (IBD). In an animal model a high production of HMGB1 by intestinal mucosal cells was found in feces of animals with intestinal infection, and the local changes were reflected also at systemic level (Splichalova and Splichal 2012). It was also suggested that the fecal HMGB1 could serve as a novel marker for intestinal inflammation and a noninvasive method of clinical evaluation of the severity of enteric infections.

A disrupted composition of intestinal flora was shown in autism. It is believed that it supports a chronic low-grade inflammation, resulting in an increased permeability of the intestinal wall. Substances produced by the immune cells upon GI inflammation enter the circulation and they may cross the blood-brain barrier, with consequences on the brain functions, including behavior (Coury et al. 2012, de Theije et al. 2011). There is evidence that also HMGB1 can cross the blood-brain barrier, thus the brain cells may be exposed not only to HMGB1 released in the brain, but also of the peripheral origin (e.g. released by intestinal mucosa) (Fang et al. 2012).

At present time possible clinical targeting of HMGB1 is being explored. Treatment with inhibitors of HMGB1 activity has been proven effective in reduction of the inflammatory activity in a wide range of preclinical disease models (Yang and Tracey 2010).

In the present study an association between plasma levels of HMGB1 and severity of GI symptoms was shown, however some limitations of the study need to be mentioned. They include a small sample size. Also, the diagnosis of an autism spectrum disorder was made by a qualified medical professional prior to enrolment in the study, but there was no additional verification, also for the control neurotypical children no verification of possible ASD symptoms was made beyond the parental report. Data about severity of autism are not available in this study, therefore possible interactions of HMGB1 levels, GI disorders, and behavioral characteristics of the individuals could not be analyzed. Another limitation is the involvement of siblings of the ASD cases into the 
control group, since this population displays different values of some biomarkers than the non-related controls. However, in the presented study, the HMGB1 levels and prevalence of GI symptoms of siblings did not significantly differ from the non-related subjects.

In order to clarify the role of this molecule in the pathomechanisms of autistic disorder, and to reveal if HMGB1 could be a reliable inflammatory marker explaining a link between the inflammatory process, the severity of GI symptoms and/or behavioral autistic traits more larger-scale studies need to be performed.

\section{Conflict of Interest}

There is no conflict of interest.

\section{Acknowledgements}

The project was supported by the grants APVV 0254-11 and APVV 0253-10. We wish to thank the individuals and families who graciously participated in this study.

\section{References}

ADAMS JB, JOHANSEN LJ, POWELL LD, QUIG D, RUBIN RA: Gastrointestinal flora and gastrointestinal status in children with autism - comparisons to typical children and correlation with autism severity. $B M C$ Gastroenterol 11: 22, 2011.

CENTERS FOR DISEASE CONTROL AND PREVENTION APA: Prevalence of autism spectrum disorders - Autism and Developmental Disabilities Monitoring Network, 14 sites, United States, 2008. MMWR Surveill Summ 61: $1-19,2012$.

COURY DL, ASHWOOD P, FASANO A, FUCHS G, GERAGHTY M, KAUL A, MAWE G, PATTERSON P, JONES NE: Gastrointestinal conditions in children with autism spectrum disorder: developing a research agenda. Pediatrics 130 (Suppl 2): S160-S168, 2012.

DE THEIJE CG, WU J, DA SILVA SL, KAMPHUIS PJ, GARSSEN J, KORTE SM, KRANEVELD AD: Pathways underlying the gut-to-brain connection in autism spectrum disorders as future targets for disease management. Eur J Pharmacol 668 (Suppl 1): S70-S80, 2011.

DEPINO AM: Peripheral and central inflammation in autism spectrum disorders. Mol Cell Neurosci 53: 69-76, 2013.

ELSABBAGH M, DIVAN G, KOH YJ, KIM YS, KAUCHALI S, MARCIN C, MONTIEL-NAVA C, PATEL V, PAULA CS, WANG C, YASAMY MT, FOMBONNE E: Global prevalence of autism and other pervasive developmental disorders. Autism Res 5: 160-179, 2012.

EMANUELE E, BOSO M, BRONDINO N, PIETRA S, BARALE F, UCELLI DI NEMI S, POLITI P: Increased serum levels of high mobility group box 1 protein in patients with autistic disorder. Prog Neuropsychopharmacol Biol Psychiatry 34: 681-683, 2010.

FANG P, SCHACHNER M, SHEN YQ: HMGB1 in development and diseases of the central nervous system. Mol Neurobiol 45: 499-506, 2012.

GOINES PE, ASHWOOD P: Cytokine dysregulation in autism spectrum disorders (ASD): possible role of the environment. Neurotoxicol Teratol 36: 67-81, 2013.

HALLMAYER J, CLEVELAND S, TORRES A, PHILLIPS J, COHEN B, TORIGOE T, MILLER J, FEDELE A, COLLINS J, SMITH K, LOTSPEICH L, CROEN LA, OZONOFF S, LAJONCHERE C, GRETHER JK, RISCH N: Genetic heritability and shared environmental factors among twin pairs with autism. Arch Gen Psychiatry 68: 1095-1102, 2011.

NAGLOVA H, BUCOVA M: HMGB1 and its physiological and pathological roles. Bratisl Lek Listy 113: 163-171, 2012.

NORIEGA DB, SAVELKOUL HF: Immune dysregulation in autism spectrum disorder. Eur J Pediatr 173: 33-43, 2014.

ONORE C, CAREAGA M, ASHWOOD P: The role of immune dysfunction in the pathophysiology of autism. Brain Behav Immun 26: 383-392, 2012.

ROSSIGNOL DA, FRYE RE: A review of research trends in physiological abnormalities in autism spectrum disorders: immune dysregulation, inflammation, oxidative stress, mitochondrial dysfunction and environmental toxicant exposures. Mol Psychiatry 17: 389-401, 2012. 
RUSSO AJ: Decreased epidermal growth factor (EGF) associated with HMGB1 and increased hyperactivity in children with autism. Biomark Insights 8: 35-41, 2013.

SAPPINGTON PL, YANG R, YANG H, TRACEY KJ, DELUDE RL, FINK MP: HMGB1 B box increases the permeability of Caco-2 enterocytic monolayers and impairs intestinal barrier function in mice. Gastroenterology 123: 790-802, 2002.

SPLICHALOVA A, SPLICHAL I: Local and systemic occurrences of HMGB1 in gnotobiotic piglets infected with E. coli O55 are related to bacterial translocation and inflammatory cytokines. Cytokine 60: 597-600, 2012.

VITALI R, STRONATI L, NEGRONI A, DI NARDO G, PIERDOMENICO M, DEL GIUDICE E, ROSSI P, CUCCHIARA S: Fecal HMGB1 is a novel marker of intestinal mucosal inflammation in pediatric inflammatory bowel disease. Am J Gastroenterol 106: 2029-2040, 2011.

YANG H, TRACEY KJ: Targeting HMGB1 in inflammation. Biochim Biophys Acta 1799: 149-156, 2010. 